\title{
XEN Gel Staining with Trypan Blue: A Simple and Effective Technique to Improve Device Visualization during Implantation
}

\author{
Vincenzo Ramovecchi ${ }^{1}$, Fabrizio Franco ${ }^{2}$, Giuseppe Ruben Barbera ${ }^{3}$
}

\begin{abstract}
The Xen Gel Stent (Allergan, Irvine, CA, USA) is a hydrophilic collagen-based gelatin tube of $6 \mathrm{~mm}$ in length which creates a channel of communication between the anterior chamber and the subconjunctival space allowing aqueous humor outflow. XEN is an ab interno, minimally invasive, glaucoma surgery device which has a better safety profile and offers a less invasive way of lowering intraocular pressure. Unfortunately, one of its weaknesses is that visualization of the implant during surgery is difficult, and this difficulty may be exacerbated, for instance, by a subconjunctival hemorrhage. The purpose of this paper is to describe a simple and effective technique for improving device visualization (XEN $45 \mu \mathrm{m}$ ) during the implantation.

Keywords: Complications, Glaucoma, Minimally invasive glaucoma surgery, XEN implant.

Journal of Current Glaucoma Practice (2021): 10.5005/jp-journals-10078-1318
\end{abstract}

\section{INTRODUCTION}

Glaucoma is an optic neuropathy that can lead to progressive irreversible blindness. ${ }^{1}$ Usually, the first treatments are medical therapy or laser therapy. However, poor compliance, side effects, and other factors can lead to failure to control the progression and consequently surgical treatment is needed. ${ }^{2,3}$ Trabeculectomy and tube shunt drainage devices are currently considered the gold standard for surgical management of glaucoma. ${ }^{4,5}$ Recently, less invasive procedures known as minimally invasive glaucoma surgery (MIGS) have been introduced. They have more successful outcomes and fewer potential complications (e.g., hypotony or endophthalmitis) than traditional surgery. ${ }^{6,7}$ Minimally invasive glaucoma surgery devices permit aqueous outflow via three main pathways: the suprachoroidal space improves uveoscleral drainage, Schlemm's canal potentiates trabecular outflow, and the subconjunctival space opens an artificial outflow pathway. ${ }^{8}$

The Xen Gel Stent (Allergan, Irvine, CA, USA) is a hydrophilic collagen-based gelatin tube of $6 \mathrm{~mm}$ in length which creates a channel of communication between the anterior chamber and the subconjunctival space allowing aqueous humor outflow and therefore IOP reduction. ${ }^{9}$ The stent is implanted with an $a b$ interno approach and, due to its biocompatibility, does not cause foreign body reactions. ${ }^{10}$ There are three models with different inner diameters: 140,63 , and $45 \mu \mathrm{m}$, designed according to the Hagen-Poiseuille equation. The XEN Gel Stent is soft and flexible. When injected it hydrates within 1 to 2 minutes, acquiring an S-shaped curve that minimizes many of the complications seen with synthetic devices, such as migration, erosion, and corneal endothelial damage. ${ }^{10}$ Xen Gel Stent implantation can be performed as a solo procedure or combined with cataract surgery. Either way, it has been shown to significantly reduce both IOP and the need for antiglaucoma medications to a rate comparable to that of trabeculectomy, but with a better safety profile. $^{7,11}$
1Department of Ophthalmology, Ospedale di San Severino Marche e
Macerata, Italy
${ }^{2}$ Department of AOU Careggi, SOD Oculistica, Firenze, Toscana, Italy
${ }^{3}$ Department of Ophthalmology, AOU Careggi, Florence, Tuscany, Italy
Corresponding Author: Fabrizio Franco, Department of AOU Careggi,
SOD Oculistica, Firenze, Toscana, Italy, Phone: +91 3337743174, e-mail:
fabriziogsfranco@gmail.com

How to cite this article: Ramovecchi V, Franco F, Barbera GR. XEN Gel Staining with Trypan Blue: A Simple and Effective Technique to Improve Device Visualization during Implantation. J Curr Glaucoma Pract 2021;15(3):161-163.

Source of support: University of Florence, Department NEUROFARBA, Eye Clinic, Florence, Italy

Conflict of interest: None

\section{Surgical Technique}

To improve the visualization of the device during implantation, we stained the XEN $45 \mu \mathrm{m}$ device with Trypan Blue 0.1\% (Oftal Blue, Alfa Intes) (Fig. 1). Injecting mitomycin C (MMC) can cause subconjunctival hemorrhage, making it difficult to see the device in the target area. Xen staining avoids this problem and improves visibility. Staining also makes the Xen device more easily distinguishable from the surrounding tissues, and checking for correct positioning is thus facilitated (Fig. 2). We placed a 23-g cannula on the syringe containing the Trypan Blue: then we inserted the cannula into the XEN injector. We then gently injected the Trypan Blue to obtain the XEN staining. Now we were ready to implant. After topical anesthesia, landmarks were drawn to mark a target area of $3 \times 3 \mathrm{~mm}$ in the superior-nasal conjunctiva before XEN positioning. Another area, $3 \mathrm{~mm}$ away from the first, was then marked in the posterior fornix as the MMC injection site. A 27-gauge needle was then used to inject $0.1 \mathrm{cc}$ of MMC $0.01 \%$ (Galenic

() The Author(s). 2021 Open Access This article is distributed under the terms of the Creative Commons Attribution 4.0 International License (https://creativecommons. org/licenses/by-nc/4.0/), which permits unrestricted use, distribution, and non-commercial reproduction in any medium, provided you give appropriate credit to the original author(s) and the source, provide a link to the Creative Commons license, and indicate if changes were made. The Creative Commons Public Domain Dedication waiver (http://creativecommons.org/publicdomain/zero/1.0/) applies to the data made available in this article, unless otherwise stated. 


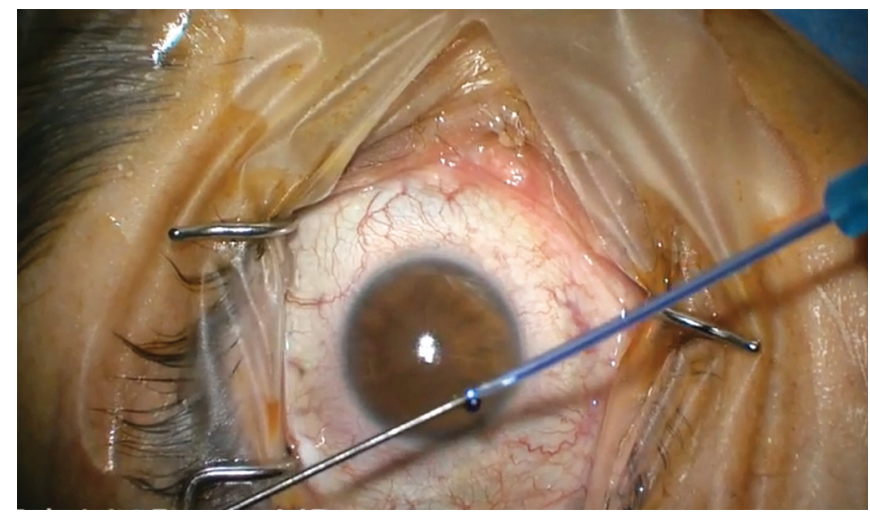

Fig. 1: Staining XEN 45 with trypan blue

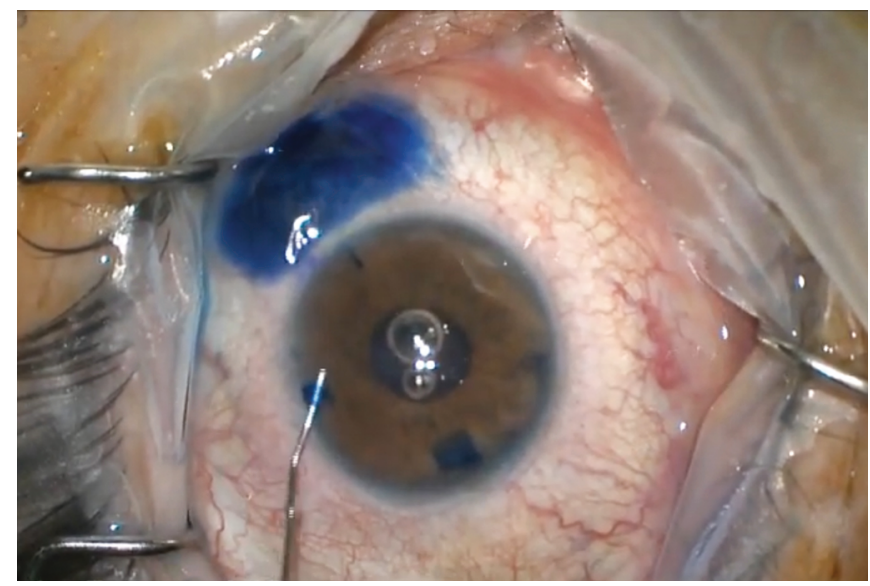

Fig. 3: Priming of the bleb

preparation from the hospital pharmacy) subconjunctivally. Corneal tunnels were created and the iridocorneal angle was visualized with a gonio-lens. After filling the anterior chamber first with lidocaine $1 \%$ (Monico) and then with viscoelastic (Johnson \& Johnson Vision Healon GV Pro), a 27-gauge preloaded injector was inserted through the corneal tunnel in the inferior-temporal quadrant. The XEN 45 stent was then placed superior-nasally in the ideal position under gonioscopic view. Finally, the viscoelastic was withdrawn from the anterior chamber and Trypan Blue $0.1 \%$ was injected in the anterior chamber with a 30-gauge needle to prime the bleb, directed at the nasal angle, where it was allowed to remain for at least 30 seconds, after which the anterior chamber was rinsed with balanced saline solution. The "priming of the bleb" makes it possible to check that the device is functioning and placed correctly (Fig. 3). We used the blue dye to better highlight the bleb. The day after surgery, no trypan remains below the bleb or in the anterior chamber. Surgery is concluded with hydro-suture of corneal incisions.

\section{Discussion}

A critical point in the surgical technique is the final placement of the XEN device. Intraoperative complications can occur: ${ }^{12,13}$ misplacement during the first attempt; posterior placement of the implant, especially through the ciliary body, causing bleeding and hypotony; anterior chamber and subconjunctival bleeding possibly caused by injecting MMC. Consequently, it is very important to find instruments that facilitate visualization, improve the contrast

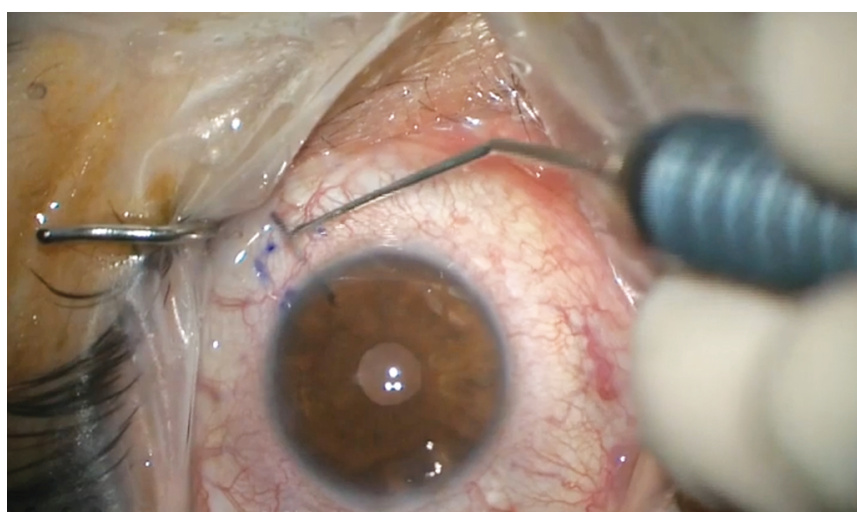

Fig. 2: Checking for correct positioning of the device

between the XEN device and the surrounding tissues, and simplify checking for correct positioning. Such tools would be really useful when visibility is compromised by complications.

Trypan blue has been demonstrated to have great potential as a surgical agent. Safe and easy to use, it has made many surgical procedures safer and easier to perform. ${ }^{14}$ Besides its application in cataract surgery, corneal transplantation, and ERM removal, it has many applications in glaucoma procedures. ${ }^{15}$ Trypan blue has already been described in evaluations of the patency of the bleb filtering and drainage device; ${ }^{16}$ it highlights the exact location for goniotomy, trabeculectomy, and XEN placement, as it selectively stains the trabecular meshwork. ${ }^{17}$ Besides its application in the initial surgery, it has also been described as assisting the needling technique for early bleb failure after XEN implantation. ${ }^{18}$ Trypan blue is safe for both corneal and trabecular meshwork cells. ${ }^{11}$ Moreover, surgeons are already familiar with its use and it is also readily available.

\section{Conclusion}

The success of XEN implantation relies critically on visibility, and because trypan blue makes the device more visible, this stain can be an especially useful tool. To the best of our knowledge, this is the first report of a surgical technique involving the staining of XEN gel with trypan blue. We hope this new technique might make a small contribution to the still novel field of MIGS.

\section{ACKnowledgment}

We thank Mr Fabrizio Marchesani for the assistance in the operating room and for the support in the creation and execution of this new staining technique.

\section{References}

1. Tham YC, Li X, Wong TY, et al. Global prevalence of glaucoma and projections of glaucoma burden through 2040: a systematic review and meta-analysis. Ophthalmology 2014;121(11):2081-2090.

2. Newman-Casey PA, Robin AL, Blachley $\mathrm{T}$, et al. The most common barriers to glaucoma medication adherence: a cross sectional survey. Ophtalmology 2015;122(7):1308-1316.

3. Lemij HG, Hoevenaars JG, van der Windt C, et al. Patient satisfaction with glaucoma therapy: reality or myth? Clin Ophthalmol 2015;9:785793.

4. Fernández S, Pardiñas N, Laliena JL, et al. Long-term tensional results after trabeculectomy. A comparative study among types of glaucoma and previous medical treatment. Arch Soc Esp Oftalmol 2009;84(7):345-352. 
5. Ansari E. An update on implants for minimally invasive glaucoma surgery (MIGS). Ophthalmol Ther 2017;6(2):233-241.

6. Kerr NM, Wang J, Barton K. Minimally invasive glaucoma surgery as primary stand-alone surgery for glaucoma. Clin Exp Ophthalmol 2017;45(4):393-400.

7. Marcos Parra MT, Salinas López JA, López Grau NS, et al. XEN implant device versus trabeculectomy, either alone or in combination with phacoemulsification, in open-angle glaucoma patients. Graefes Arch Clin Exp Ophthalmol 2019;257(8):1741-1750.

8. Lavia C, Dallorto L, Maule M, et al. Minimally-invasive glaucoma surgeries (MIGS) for open angle glaucoma: a systematic review and meta-analysis. PLoS One 2017;12(8):e0183142.

9. De Gregorio A, Pedrotti E, Stevan G, et al. XEN glaucoma treatment system in the management of refractory glaucomas: a short review on trial data and potential role in clinical practice. Clin Ophthalmol 2018;12:773-782.

10. Lewis R. Ab-interno approach to the subconjunctival space using a collage glaucoma stent. J cataract refract sur 2014;40: 1301-1306.

11. Hohberger B, Welge-Lüßen UC, Lämmer R. MIGS: therapeutic success of combined Xen gel stent implantation with cataract surgery. Graefes Arch Clin Exp Ophthalmol 2018;256(3): 621-625.

12. Gupta C, Mathews D. XEN ${ }^{\circledR}$ stent complications: a case series. BMC Ophthalmol 2019;19(1):253. DOI: 10.1186/s12886-019-1267-y.

13. Sng CCA, Chew PTK, Htoon HM, et al. Case series of combined XEN implantation and phacoemulsification in Chinese eyes: one-year outcomes. Adv Ther 2019;36(12):3519-3529.

14. Tsaousis KT, Kopsachilis N, Tsinopoulos IT, et al. Time-dependent morphological alterations and viability of cultured human trabecular cells after exposure to Trypan blue. Clin Exp Ophthalmol 2013;41:484490.

15. Jhanji V, Chan E, Das S, et al. Trypan blue dye for anterior segment surgeries. Eye 2011;25:1113-1120.

16. Grover DS, Fellman RL. Confirming and establishing patency of glaucoma drainage devices using trypan blue. J Glaucoma 2013;22:e1-e2.

17. Rodrigues EB, Costa EF, Penha FM, et al. The use of vital dyes in ocular surgery. Surv Ophthalmol 2009;54(5):576-617.

18. Ferreira NP, Pinto JM, Teixeira F, et al. XEN gel stent early failure-dyeenhanced Ab-externo revision. J Curr Glaucoma Pract 2018;12(3):139141. 